\title{
Efeito de Duas Cargas Animais em Campo Nativo e de Duas Idades à Desmama no Desempenho de Vacas de Corte Primíparas ${ }^{1}$
}

\author{
José Inácio Braccini Fagundes², José Fernando Piva Lobato ${ }^{3}$, Flávio Schramm Schenkel ${ }^{4}$
}

\begin{abstract}
RESUMO - A investigação foi realizada para avaliar os efeitos de duas cargas animais (CA280=280 kg PV/ha e CA360=360 kg $\mathrm{PV} / \mathrm{ha}$ ) e de duas idades de desmama dos bezerros ( $\mathrm{DC}=$ Desmama convencional aos sete meses de idade e $\mathrm{DP}=\mathrm{Desmama}$ precoce aos três/ quatro meses) no comportamento reprodutivo de vacas de corte primíparas de duas composições raciais ( $1 / 2$ Nelore $1 / 2$ Hereford e $1 / 4$ Nelore $3 / 4$ Hereford). Foram avaliados os ganhos de peso médios diários (GMD) do pós-parto à data da desmama convencional, durante o acasalamento, da desmama precoce à desmama convencional (GPC), a condição corporal (CC) ao início do acasalamento, ao final do acasalamento e na data da desmama convencional, a taxa de prenhez (TP) e o intervalode partos (IEP). As vacas conduzidas na CA280 tiveram significativamente maiores GMD do que vacas na CA360 nos três períodos estudados. Vacas submetidas ao tratamento de desmama precoce tiveram GPC maiores que vacas submetidas à desmama convencional ( $0,539 \mathrm{vs.} 0,257 \mathrm{~kg} / \mathrm{dia}$, respectivamente). Vacas da CA280 tiveram significativamente maiores condições corporais do início do acasalamento à data da desmama convencional do que vacas da CA360, sendo que vacas submetidas à desmama precoce apresentaram condição corporal na data da desmama convencional superior àquela das vacas da desmama convencional sob ambas cargas animais (4,33 vs. 3,68 para CA280 e 3,14 vs. 2,92 para CA360). A composição racial das vacas não influenciou significativamente o GMD e a condição corporal das vacas no período experimental. A TP da CA280 (67,56\%) foi significativamente superior à da CA360 (22,56\%), bem como o seu IEP foi significativamente inferior ao da CA360 (395,4 vs. 409,9 dias). Portanto, a carga animal menor permitiu que as vacas apresentassem maiores recuperações de peso e condição corporal no pós-parto, determinando melhores taxas de reconcepção e menores intervalos de partos.
\end{abstract}

Palavras-chave: desempenho reprodutivo, idade de desmama, vacas primíparas

\section{Effect of Two Stocking Rates on Natural Pasture and Two Weaning Ages on Primiparous Beef Cows Performance}

\begin{abstract}
The investigation was carried out to evaluate the effects of two stocking rates (CA280 $=280 \mathrm{~kg} \mathrm{LW} / \mathrm{ha}$ and CA=360= $360 \mathrm{~kg} \mathrm{LW} / \mathrm{ha}$ ) and two weaning ages of calves ( $\mathrm{DC}=$ Conventional weaning at seven months of age and DP= Early weaning at three/four months of age) on reproductive performance of primiparous beef cows of two breed compositions (1/2 Nelore $1 / 2$ Hereford and $1 / 4$ Nelore $3 / 4$ Hereford). Average daily weight gain (GMD) from calving to DC, during the mating period, and in the period from DP to DC (GPC), the body condition score (CC) at beginning and at the end of mating period and at DC, the pregnancy rate (TP), and the calving interval (IEP) of the cows were evaluated. Cows managed under CA280 had significantly higher GMD than cows under CA360 in all three periods considered. Cows submitted to DP had higher GPC $(\mathrm{P}<0.01)$ than cows submitted to DC $(0.539 \mathrm{vs.} 0.257 \mathrm{~kg} /$ day, respectively $)$. Cows under CA280 had signicantly higher CC from the beginning of the mating period to the date of DC than cows under CA360, while cows submitted to DP showed higher CC at the date of DC than cows submitted to DC under both stocking rates (4.33 vs. 3.68 for CA280 and 3.14 vs. 2.92 for CA360). The breed composition of the cows did not significantly influence on GMD and CC of the cows over the experimental period. The TP of cows under CA280 (67.56\%) was significantly better than cows under CA360 (22.56\%), as well as their IEP were significantly lower than cows under CA360 (395.4 vs. 409.9 days). Therefore, the lowest stocking rate allowed cows to better recover their weight and body condition after calving, causing improvement of conception rate and calving interval.
\end{abstract}

Key Words: primiparous crossbred cows, reproductive performance, weaning age

\section{Introdução}

A carga animal, índice que expressa a quantidade de kg de peso vivo (PV) por unidade de superfície (Mott, 1980), é o fator determinante da produção por animal e por área na pecuária em pastoreio (Harlan, 1958).

Lobato (1985) relata que o fator mais limitante na produção pecuária é a deficiência nutricional do campo nativo no período hibernal, somando a ausên-

\footnotetext{
1 Parte da dissertação de Mestrado do primeiro autor - Departamento de Zootecnia, UFRGS, bolsista CNPq.

${ }^{2}$ Med. Vet., M.Sc. em Zootecnia - UFRGS, Porto Alegre - RS. E.mail: quino@bnet.com.br

3 Prof. Adjunto IV, Dep. de Zootecnia-UFRGS, Bolsista CNPq, Porto Alegre-RS, C.Postal. 776-90.001-970. E.mail: lobato@orion.ufrgs.br

${ }^{4}$ Pesquisador associado, Universidade de Guelph, Guelph, Canadá. E.mail: schenkel@uoguelph.ca
} 
cia de práticas de manejo que permitiriam uma melhor e mais eficiente utilização do mesmo.

Mudanças na carga animal têm reflexos sobre o desempenho reprodutivo. No Rio Grande do Sul, como a exploração e a produtividade dos rebanhos de cria são feitas sobre os campos nativos, trabalhos têm sido conduzidos em campo nativo com o objetivo de identificar para os produtores as cargas animais que possibilitem melhores desempenhos reprodutivos. Quadros \& Lobato (1996) observaram, para cargas animais de 320 e $240 \mathrm{~kg} \mathrm{PV} / \mathrm{ha}$, taxas de prenhez de 84,2 e $96,8 \%$, respectivamente. Gottschall \& Lobato (1996), em trabalho com vacas primíparas com baixo peso e condição corporal (CC) ao parto, submetidas a três cargas (280, 320 e $360 \mathrm{~kg}$ PV/ha), obtiveram repetições de prenhez de 8,$5 ; 10,4$ e $0 \%$, respectivamente. Simeone \& Lobato (1996) obtiveram repetições de prenhez de 25 e $50 \%$ para cargas de 340 e $240 \mathrm{~kg}$ PV/ha, respectivamente. Magalhães \& Lobato (1991), trabalhando com 180 e $315 \mathrm{Kg}$ PV/ha, obtiveram, na carga mais baixa, melhor resposta reprodutiva (74\% de prenhez) do que na carga mais alta (59\%).

Hart et al. (1988) relatam a existência de diferenças de produção entre diferentes categorias animais quando manejadas em uma dada carga animal. Esses autores afirmam serem as vacas mais sensíveis às mudanças de carga animal do que animais em crescimento ou bezerros desmamados.

A carga animal deve ser relacionada à oferta alimentar disponível aos animais, sendo que cargas mais altas associam-se a níveis nutricionais mais baixos, observando-se menores condições corporais de vacas nos diferentes momentos do ciclo produtivo. Objetivou-se, com este trabalho, avaliar o efeito de duas cargas animais e de duas idades à desmama dos bezerros, em campo nativo, sobre o ganho médio diário (GMD), a variação da condição corporal (CC), o desempenho reprodutivo e o intervalo de partos (IEP), de vacas Braford, com diferentes composições raciais.

\section{Material e Métodos}

Foram utilizadas 78 vacas primíparas da raça Braford, de composições raciais $1 / 2$ Nelore $1 / 2$ Hereford e $1 / 4$ Nelore $3 / 4$ Hereford, distribuídas em dois potreiros, correspondendo cada um ao tratamento de carga animal. Ambos potreiros apresentavam semelhante distribuição de aguadas, sombras e saleiros, sendo também de mesma topografia e formação campestre, localizados um ao lado do outro, separados por aramado convencional.

A disponibilidade forrageira foi avaliada usando-se dois métodos: Método Comparativo (Haydock \& Shaw, 1975) e método do quadrado, sendo feitos cinco cortes de $0,25 \mathrm{~m}^{2}$ rentes ao solo, sendo escolhido e pontuado cada corte desde a disponibilidade mínima (1), até a máxima (5), e também 40 avaliações visuais ao acaso e pontuadas de 1 a 5 , de acordo com os cortes previamente realizados, nos dois potreiros, a cada 28 dias.

Previamente à alocação dos animais nos respectivos tratamentos, a disponibilidade de forragem inicial dos dois potreiros foi equalizada, com a utilização de roçadeira e pastejo com animais. A disponibilidade inicial foi de 2.768 e $2.852 \mathrm{~kg} \mathrm{MS} / \mathrm{ha}$, para os tratamentos CA280 e CA360, respectivamente. Os animais foram alocados nos potreiros em 21/08/1999, onde permaneceram até o final do experimento (13/04/2000).

Os tratamentos foram os seguintes, dispostos no delineamento completamente casualizado em arranjo fatorial:

\section{Carga animal}

- CA280 - potreiro com carga animal de $280 \mathrm{~kg}$ $\mathrm{PV} / \mathrm{ha}$ (ocupado por 37 vacas).

- CA360 - potreiro com carga animal de $360 \mathrm{~kg}$ $\mathrm{PV} / \mathrm{ha}$ (ocupado por 41 vacas).

\section{Idade de desmama}

- Desmama precoce (DP) - bezerros desmamados em 13/01/2000, com um peso vivo mínimo de $70 \mathrm{~kg}$ e peso médio de $110,80 \mathrm{~kg}$.

- Desmama convencional (DC) - bezerros desmamados em 09/04/2000 (aproximadamente sete meses), com peso médio de $128,92 \mathrm{~kg}$.

O primeiro período de parição ocorreu de 21/08/99 a 30/10/99. Vacas e bezerros foram pesados até 24 horas após o parto, a fim de avaliar o peso pós-parto da vaca (PP) e o peso ao nascer do bezerro (PN). Posteriormente, vacas e bezerros foram pesados a cada 28 dias, sendo também avaliada a condição corporal (CC) das vacas (escala de 1 a 5 , sendo $1=$ magra e 5= gorda, conforme Lowman et al., 1976), até a data da desmama dos bezerros da desmama convencional (13/04/00).

O período de acasalamento foi de 15/11/99 a $15 / 02 / 00$, sendo usados dois touros em cada potreiro, previamente submetidos ao exame andrológico. O diagnóstico de gestação foi realizado por palpação retal em 24/04/00.

As variáveis de resposta contínua foram analisa- 
das pela análise da variância, considerando número desigual de repetições, por meio do programa estatístico SAS (SAS, 1990). Os procedimentos Proc GLM e Proc Mixed foram utilizados, sendo o segundo aplicado para os dados de medidas repetidas no mesmo animal (unidade experimental). Diferenças entre múltiplas médias foram testadas pelo teste de Tukey (Steel \& Torrie, 1989).

A condição corporal (CC) das vacas ao longo do período experimental foi analisada por intermédio do Proc Mixed dos SAS, assumindo ter distribuição contínua, usando o seguinte modelo:

$$
\begin{gathered}
Y_{i j k l m}=\mu+\mathrm{CA}_{\mathrm{i}}+\mathrm{CR}_{\mathrm{j}}+\mathrm{CA}^{*} \mathrm{CR}_{\mathrm{ij}}+\mathrm{Va}_{\mathrm{k}}: \mathrm{CA}^{*} \mathrm{CR} \\
+\mathrm{S}_{1}+\beta_{1} \mathrm{Mês}_{\mathrm{m}}: \mathrm{CA}+\beta_{2} \mathrm{Mêsm} 2: \mathrm{CA}+\beta_{3} \mathrm{DJP}_{\mathrm{k}}+ \\
\beta_{3} \mathrm{DJP}_{\mathrm{k}}{ }^{2}+\mathrm{e}_{\mathrm{ijklm}}
\end{gathered}
$$

em que: $\mathrm{Y}_{\mathrm{ijklm}}=\mathrm{CC}$ da k-ésima vaca no m-ésimo mês, pertencente à i-ésima carga animal e j-ésima composição racial; $\mu=$ média geral; $\mathrm{CA}_{\mathrm{i}}=$ efeito fixo da i-ésima carga animal; $\mathrm{CR}_{\mathrm{j}}=$ efeito fixo da j-ésima composição racial; $\mathrm{CA} * \mathrm{CR}_{\mathrm{ij}}=$ efeito fixo da interação entre i-ésima carga animal e j-ésima composição racial; $\mathrm{Va}_{\mathrm{k}}: \mathrm{CA} * \mathrm{CR}=$ efeito aleatório da k-ésima vaca aninhada em carga animal e composição racial; $\mathrm{S}_{1}=$ efeito fixo do l-ésimo sexo do bezerro; Mês $_{\mathrm{m}}=$ M-ésimo mês de medida; $\beta_{1}: \mathrm{CA}=$ coeficiente de regressão linear aninhado em carga animal; $\beta_{2}: C A=$ coeficiente de regressão quadrático aninhado em carga animal; $\mathrm{DJP}_{\mathrm{k}}=$ data juliana do parto da k-ésima vaca; $\beta_{3}=$ coeficiente de regressão linear; $\beta_{4}=$ coeficiente de regressão quadrático; $\mathrm{e}_{\mathrm{ijklm}}=$ efeito residual aleatório associado à observação $y_{\mathrm{ijklm}}$.

Para a análise da condição corporal das vacas no início do acasalamento e na data da desmama precoce, o modelo descrito acima incluiu o efeito classificatório do mês aninhado em carga animal, em vez da regressão. Os efeitos foram obtidos por contraste entre as soluções para as cargas animais, para o mês apropriado.

Para a análise da condição corporal das vacas no período do início do acasalamento e a data da desmama convencional, o modelo descrito anteriormente incluiu o efeito da idade de desmama e as interações duplas com carga animal e composição racial, bem como o efeito do mês como classificatório, aninhado em carga animal e idade de desmama, em vez da regressão.

O ganhos médios diários de peso (GMD) das vacas do pós-parto à desmama convencional e da desmama precoce à desmama convencional foram analisados por meio do Proc GLM dos SAS, usando o seguinte modelo:

$$
\begin{gathered}
Y_{i j k l m n}=\mu+C A_{i}+I_{j}+C A * I_{i j}+C_{k}+S_{1}+ \\
P_{m}+\beta_{1} D P_{n}+\beta_{2} D P_{n}{ }^{2}+e_{i j k l m n}
\end{gathered}
$$

em que: $y_{i j k l m n}=$ GMD da n-ésima vaca, pertencente à i-ésima e j-ésima idade de desmama e da k-ésima composição racial; $\mu=$ média geral; $\mathrm{CA}_{\mathrm{i}}=$ efeito fixo da i-ésima carga animal; $\mathrm{ID}_{\mathrm{j}}=$ efeito fixo da j-ésima idade de desmama; $\mathrm{CA} * \mathrm{IDij}=$ efeito fixo da interação entre i-ésima carga animal e j-ésima idade de desmama; $\mathrm{CR}_{\mathrm{k}}=$ efeito fixo da k-ésima composição racial; $\mathrm{S}_{1}=$ efeito fixo do l-ésimo sexo do bezerro; $\mathrm{P}_{\mathrm{m}}=$ efeito fixo da m-ésimo estado gestacional (prenhe ou vazia); $\operatorname{DJP}_{\mathrm{n}}=$ data juliana do parto da n-ésima vaca; $\beta_{1}=$ coeficiente de regressão linear; $\beta_{2}=$ coeficiente de regressão quadrático; $\mathrm{e}_{\mathrm{ijklmn}}=$ efeito residual aleatório associado à observação yi ${ }_{\text {jklmn }}$.

Para a análise do GMD das vacas durante o acasalamento, o modelo anterior não incluiu o efeito da prenhez.

As análises do intervalo de partos (IEP) foram realizadas por meio do Proc GLM, empregando o seguinte modelo:

$$
\mathrm{Y}_{\mathrm{ijkl}}=\mu+\mathrm{CA}_{\mathrm{i}}+\mathrm{ID}_{\mathrm{j}}+\mathrm{CR}_{\mathrm{k}}+\beta_{1} \mathrm{DJP}_{1}+\mathrm{e}_{\mathrm{ijkl}}
$$

em que: $Y_{i j k}=$ IEP da l-ésima vaca, pertencente à i-ésima carga animal, submetida a j-ésima idade de desmama, com a k-ésima composição racial; $\mu=$ média geral; $\mathrm{CA}_{\mathrm{i}}=$ efeito fixo da i-ésima carga animal; $\mathrm{ID}_{\mathrm{j}}=$ efeito fixo da j-ésima idade de desmama; $\mathrm{CR}_{\mathrm{k}}=$ efeito fixo da k-ésima composição racial; $\mathrm{DJP}_{1}=$ data juliana do parto da 1-ésima vaca; $\beta_{1}=$ coeficiente de regressão linear; $\mathrm{e}_{\mathrm{ijkl}}=$ efeito residual aleatório associado à observação $\mathrm{y}_{\mathrm{ijkl}}$.

Os efeitos dos tratamentos sobre a percentagem de prenhez foram analisados pelo teste de qui-quadrado (Steel \& Torrie, 1989), por meio do Proc Freq do SAS com a opção "cmh", para ajustar para outras fontes de variação, que não aquelas da tabela de contingência, usando o seguinte modelo:

$$
\mathrm{Y}_{\mathrm{ijkl}}=\mathrm{CA}_{\mathrm{i}}+\mathrm{ID}_{\mathrm{j}}+\mathrm{CR}_{\mathrm{k}}+\mathrm{e}_{\mathrm{ijkl}}
$$

em que: $Y_{i j k l}=$ prenhez (prenha ou vazia) da l-ésima vaca, pertencente à i-ésima carga animal e a j-ésima idade de desmama, da k-ésima composição racial; $\mathrm{CA}_{\mathrm{i}}=$ efeito fixoda i-ésima carga animal; $\mathrm{ID}_{\mathrm{j}}=$ efeito fixo da j-ésima idade de desmama; $\mathrm{CR}_{\mathrm{k}}=$ efeito fixo da k-ésima composição racial; $\mathrm{e}_{\mathrm{ijkl}}=$ efeito residual aleatório associado à observação $\mathrm{y}_{\mathrm{ijk} k}$.

$\mathrm{O}$ efeito da carga animal sobre a disponibilidade de forragem, medida pelo método comparativo e pelo método do quadrado, foi analisado usando o seguinte modelo, por meio do Proc GLM do SAS: 


$$
\mathrm{Y}_{\mathrm{ij}}=\mu+\mathrm{CA}_{\mathrm{i}}+\mathrm{Mês}_{\mathrm{j}}+\mathrm{e}_{\mathrm{ij}}
$$

em que: $Y_{i j}=$ medida de disponibilidade de forragem na i-ésima carga animal, tomada no j-ésimo mês; $\mu=$ média geral; $\mathrm{CA}_{\mathrm{i}}=$ efeito fixo da i-ésima carga animal; $\mathrm{Mes}_{\mathrm{j}}=$ efeito fixo do $\mathrm{j}$-ésimo mês; $\mathrm{e}_{\mathrm{ij}}=$ efeito residual aleatório associado à observação $\mathrm{y}_{\mathrm{ij}}$.

O efeito da carga animal sobre a altura do pasto foi analisada usando o seguinte modelo, por meio do Proc GLM do SAS:

$$
\mathrm{Y}_{\mathrm{ijk}}=\mu+\mathrm{CA}_{\mathrm{i}}+\mathrm{Mês}_{\mathrm{j}}+\mathrm{CA} \mathrm{CAês}_{\mathrm{ij}}+\mathrm{e}_{\mathrm{ijk}}
$$

em que: $\mathrm{Y}_{\mathrm{ijk}}=\mathrm{K}$-ésima medida de de altura de pasto na i-ésima carga animal, tomada no j-ésimo mês; $\mu=$ média geral; $\mathrm{CA}_{\mathrm{i}}=$ efeito fixo da i-ésima carga animal; Mês $=$ efeito fixo do j-ésimo mês; $\mathrm{CA}^{*} \mathrm{Mês}_{\mathrm{ij}}=$ efeito fixo da interação entre a i-ésima carga animal e o j-ésimo mês; $\mathrm{e}_{\mathrm{ijk}}=$ efeito residual aleatório associado à observação $y_{i j k}$.

\section{Resultados e Discussão}

Nas Figuras 1 e 2, são apresentadas as variações na disponibilidade de forragem e na altura da forragem, durante o período experimental, respectivamente. Houve diferença significativa $(\mathrm{P}<0,01)$ na disponibilidade de forragem, entre as duas cargas animais estudadas. A disponibilidade média ajustada para todo o período foi de 3.218 e $2.418 \mathrm{~kg} \mathrm{MS} / \mathrm{ha}$, respectivamente, para CA280e CA360. A altura média do pasto, durante o período experimental, também apresentou diferença significativa $(\mathrm{P}<0,01)$ entre as cargas animais analisadas $(5,86$ e 4,14 cm para CA280 e CA360, repectivamente). Estes resultados referentes à disponibilidade de forragem foram um fator determinante dos resultados produtivos observados nos animais.

De acordo com Hodgson et al. (1971), o menor número de animais por unidade de área permite um maior crescimento de forragem. O desempenho animal é determinado pelo valor nutritivo do pasto e pelo nível de consumo, sendo este afetado pela quantidade de forragem em oferta (Van Soest, 1982). Segundo Freitas et al. (1976), ocorrem no Rio Grande do Sul períodos críticos na disponibilidade forrageira do campo nativo, chegando nos meses de inverno a um déficit do $60 \%$ em proteína digestível e 23\% em NDT, para um bovino de $270 \mathrm{~kg}$. Isto reforça os princípios de que é impossível manter a mesma carga animal ao longo do ano, sendo necessárias cargas melhor ajustadas nos meses de maior produção forrageira, a fim de permitir melhor disponibilidade alimentar nos meses mais rigorosos.

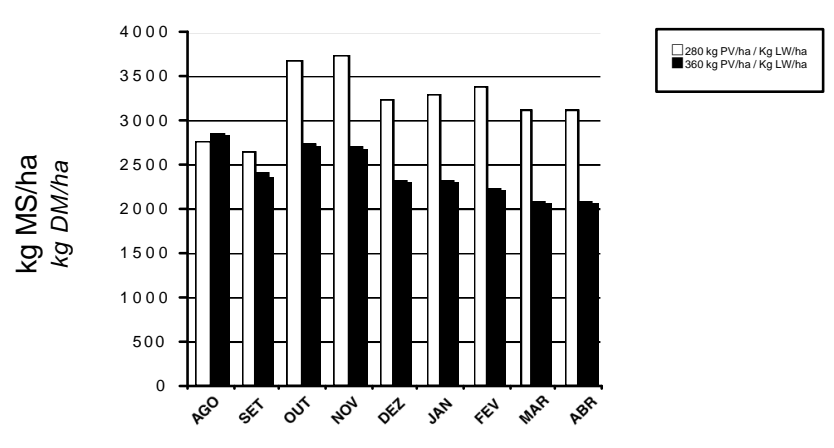

Figura 1 - Disponibilidade de forragem, durante o período experimental, conforme a carga animal.

Figure 1 - Availability of forage during the experimental period, according to the stocking rate.

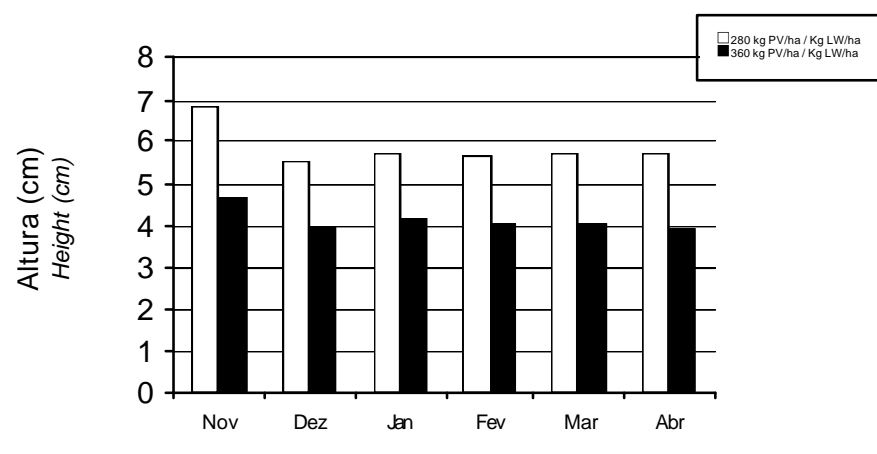

Figura 2 - Variação da altura do pasto,durante o período experimental, conforme a carga animal.

Figure 2 - Variation of the pasture height during the experimental period, according to the stocking rate.

Na Tabela 1, são apresentados os ganhos médios diários de peso e as variações de condição corporal, durante o período experimental, conforme a carga animal e a idade de desmama. A composição racial das vacas não influenciou significativamente $(\mathrm{P}>0,05)$ o GMD e a condição corporal das vacas no período experimental. A carga animal influenciou significativamente o ganho de peso das vacas do pós-parto à desmama convencional, tendo as vacas mantidas no tratamento CA280 ganho superior em relação àquelas do tratamento CA360 $(\mathrm{P}<0,05)$. Isto foi um reflexo direto da maior oferta alimentar, já demonstrada por maior disponibilidade de forragem média, durante o período analisado. Estes resultados de desempenho 
das vacas concordam com os observados por Magalhães \& Lobato (1991) e Simeone \& Lobato (1996), onde cargas mais baixas permitiram melhores ganhos de peso durante o período experimental. Quadros \& Lobato (1996) também observaram, no terço final do acasalamento, maior ganho de peso para as vacas conduzidas em menor carga animal (0,197 vs. $0,059 \mathrm{~kg} /$ dia para as cargas animais de 240 e $320 \mathrm{~kg} \mathrm{PV} / \mathrm{ha}$, respectivamente), em virtude de maior oferta alimentar para a carga animal mais baixa.

As vacas mantidas em CA280 apresentaram ganho médio diário significativamente maior $(\mathrm{P}<0,05)$, durante o período de acasalamento, do que as vacas da CA360, sendo este, novamente, o resultado da maior disponibilidade da forragem (Figura 1). Em alguns experimentos no Rio Grande do Sul, avaliando o desempenho de vacas durante o acasalamento, manejadas em diferentes cargas animais, não foram encontradas diferenças significativas no ganho de peso das vacas. Quadros \& Lobato (1996) observaram, durante os primeiros 50 dias de acasalamento (total de 108 dias), GMD de 0,581 e 0,502 kg/dia para cargas animais de 280 e $320 \mathrm{~kg}$ PV/ha (P>0,05), respectivamente. A inexistência de diferença significativa entre os ganhos foi justificada, segundo os autores, pela boa disponibilidade e qualidade da forragem disponível, até então, aos animais em ambos os tratamentos.

Gottschal \& Lobato (1993) observaram ganhos médios diários durante o acasalamento de 0,339, 0,425 e $0,335 \mathrm{~kg} / \mathrm{dia}(\mathrm{P}<0,05)$ para carga animal de 280, 320 e $360 \mathrm{~kg}$ PV/ha, respectivamente. O maior ganho observado na carga animal média foi explicado pelos autores por ter o pasto desse potreiro teor mais elevado de PB e digestibilidade da matéria orgânica. Já Simeone \& Lobato (1996) observaram ganho médio diário maior para a carga animal mais baixa $(280 \mathrm{~kg}$ $\mathrm{PV} / \mathrm{ha}=0,337 \mathrm{~kg} / \mathrm{dia} ; 340 \mathrm{~kg} \mathrm{PV} / \mathrm{ha}=0,149 \mathrm{~kg} / \mathrm{dia}$ ). Essas variações nos resultados observados pode ser explicada pelo grande número de variáveis envolvidas, como clima, solo, relevo, tipo de pastagem, diferenças entre animais, efeito do ano, entre outros.

$\mathrm{O}$ efeito da idade de desmama dos bezerros no GMD das vacas (Tabela 1), da desmama precoce à convencional e durante o acasalamento (GDA), foi significativo $(\mathrm{P}<0,05)$. Vacas submetidas à desmama precoce dos seus bezerros apresentaram GMD superiores àquelas com bezerros da desmama convencional, em todos os períodos de ganhos considerados, inclusive quando o período avaliado foi do pós-parto até a desmama convencional $(0,236$ e $0,145 \mathrm{~kg} / \mathrm{dia}$, respectivamente). Os GMD das vacas da desmama precoce à desmama convencional foram de 0,539 e $0,257 \mathrm{~kg} /$ dia, respectivamente $(\mathrm{P}<0,01)$. Segundo Monje et al. (1993), o efeito do fim da lactação sobre as vacas é impactante, diminuindo os requerimentos nutricionais das mesmas. Os requerimentos energéticos de vacas em lactação são $31 \%$ maiores para mantença e $29 \%$

Tabela 1 - Ganho de peso médio diário (GMD) das vacas do pós-parto à desmama convencional (GPD), da desmama precoce à convencional (GPC) e durante o acasalamento (GDA) e escore de condição corporal das vacas no início ( $\mathrm{CCI}$ ) e ao final (CCF) do acasalamento e na desmama convencional dos bezerros (CCD) para as cargas animais CA280 e CA360 e as desmamas convencional (DC) e precoce (DP)

Table 1 - Average cow daily weight gain (ADG) from post-partum to conventional weaning (GPD), from early weaning to conventional weaning (GPC), and during the mating period (GDA) and cow body condition score at the beginning (CCI) and at the end (CCF) of the mating period, and at conventional weaning of the calves (CCD) for stocking rates CA280 and CA360, and conventional $(C W)$ and early weaning $(E W)$

\begin{tabular}{|c|c|c|c|c|c|c|c|c|}
\hline \multirow{4}{*}{$\begin{array}{l}\text { Tratamentos } \\
\text { Treatments }\end{array}$} & \multicolumn{4}{|c|}{$\begin{array}{l}\text { GMD (kg/dia) } \\
A D G(k g / d a y)\end{array}$} & \multicolumn{4}{|c|}{$\begin{array}{l}\text { Condição corporal }(\mathrm{CC}) \\
\text { Body condition score }(B C)\end{array}$} \\
\hline & \multirow{3}{*}{$\begin{array}{l}\text { GPD } \\
G P D\end{array}$} & \multirow{3}{*}{$\begin{array}{l}\mathrm{GPC} \\
G P C\end{array}$} & \multirow{3}{*}{$\begin{array}{l}\text { GDA } \\
\text { GDA }\end{array}$} & \multirow{3}{*}{$\begin{array}{l}\text { CCI } \\
C C I\end{array}$} & \multirow{2}{*}{\multicolumn{2}{|c|}{$\begin{array}{l}\text { CCF } \\
C C F\end{array}$}} & \multirow{2}{*}{\multicolumn{2}{|c|}{$\begin{array}{l}C C D \\
C C D\end{array}$}} \\
\hline & & & & & & & & \\
\hline & & & & & $\begin{array}{l}\mathrm{DC} \\
\mathrm{CW}\end{array}$ & $\begin{array}{l}\text { DP } \\
E W\end{array}$ & $\begin{array}{l}\mathrm{DC} \\
\mathrm{CW}\end{array}$ & $\begin{array}{l}\text { DP } \\
E W\end{array}$ \\
\hline CA280 & $0,278^{\mathrm{a}}$ & $0,503^{\mathrm{a}}$ & $0,427^{\mathrm{a}}$ & $3,10^{\mathrm{a}}$ & $3,46^{\mathrm{a}}$ & $4,09 \mathrm{c}$ & $3,91^{\mathrm{a}}$ & $4,58^{\mathrm{c}}$ \\
\hline CA360 & $0,102^{\mathrm{b}}$ & $0,292^{\mathrm{b}}$ & $0,120^{\mathrm{b}}$ & $2,81^{\mathrm{b}}$ & $2,85^{b}$ & $3,01^{b}$ & $3,00^{\mathrm{b}}$ & $3,28^{\mathrm{d}}$ \\
\hline DC & $0,145^{\mathrm{B}}$ & $0,257^{\mathrm{B}}$ & $0,144^{\mathrm{B}}$ & - & - & - & - & - \\
\hline DP & $0,236^{\mathrm{A}}$ & $0,539^{\mathrm{A}}$ & $0,236^{\mathrm{A}}$ & - & - & - & - & - \\
\hline
\end{tabular}

Para GMD, médias seguidas de letras diferentes nas colunas, dentro de cada tratamento, diferem significativamente $(\mathrm{P}<0,05)$ pelo $\mathrm{F}$ teste.

Para CC, médias seguidas de letras diferentes nas colunas e linhas diferem significativamente $(P<0,05)$ pelo teste Tukey.

For ADG, means followed by different letters in the columns, within each treatment, are significantly different $(P<.05)$ by $F$ test.

For $B C$, means followed by different letters in the columns and rows are significantly different $(P<.05)$ by Tukey test. 
maiores para ganho de peso do que os correspondentes para as vacas secas.

Simeone \& Lobato (1996) também observaram maior GMD $(\mathrm{P}<0,01)$ para vacas com bezerros desmamados precocemente $(0,410 \mathrm{~kg} / \mathrm{dia})$ frente àquelas submetidas à desmama à idade convencional $(0,015 \mathrm{~kg} / \mathrm{dia})(\mathrm{P}<0,01)$. Lobato \& Barcellos $(1992)$ mediram ganho de peso superior em vacas com bezerros desmamados precocemente, frente àquelas com bezerros desmamados na época convencional (100 dias vs. 180 dias, respectivamente). Lobato et al. (2000), trabalhando com pastagens melhoradas, também observaram diferença $(\mathrm{P}<0,05)$ de $0,091 \mathrm{~kg} /$ dia superior para as vacas com bezerros desmamados precocemente.

Assim, vacas manejados em cargas animais menores apresentam GMD significativamente superiores, em razão da melhor disponibilidade alimentar. Além disso, a supressão da lactação, em virtude da desmama precoce, permitiu às vacas maior recuperação do peso vivo, devido à redução nas suas exigências, frente às vacas mantidas com seus bezerros até a idade do desmama convencional.

A condição corporal das vacas foi influenciada significativamente pela carga animal em que as vacas foram manejadas durante o experimento. No início do período de acasalamento, as vacas da CA280 apresentavam condições corporais significativamente superiores às das vacas da CA360 (3,10 e 2,81, respectivamente; $\mathrm{P}<0,01)$, coincidindo com os resultados observados por Simeone \& Lobato (1996), no mesmo período (escores de 2,08 e 1,71, para cargas de $240 \mathrm{~kg} \mathrm{PV} / \mathrm{ha}$ e $340 \mathrm{~kg} \mathrm{PV} / \mathrm{ha}$, respectivamente; $\mathrm{P}<0,05)$. Para obter bons níveis de concepção, é necessário que as vacas entrem na época de reprodução, principalmente vacas em lactação, em bom estado corporal, que deve ser mantido durante esse período (Osoro, 1986). Nicol \& Nicoll (1987) também afirmam ser a condição corporal ao início do acasalamento uma fonte de variação significativa na performance reprodutiva de vacas de corte.

Hancock et al. (1985) afirmam ser a condição corporal ao início do acasalamento uma das fases mais críticas do ciclo reprodutivo, estando muito relacionada com o desempenho reprodutivo das vacas, sendo recomendável a adequação de cargas para obtenção de melhores índices produtivos. Richards et al. (1989) observaram em vacas perdendo condição corporal no acasalamento (abaixo de 2,5 na escala 1 a 5) o cessamento da atividade lútea, tendo essa só reiniciado quando as mesmas vacas atingiram condição corporal 2,8 , ao contrário daquelas que mantiveram ou aumentaram sua condição corporal. Wright et al. (1987) estimaram que o incremento em uma unidade na condição corporal (escala 1 a 5), no pós-parto, reduz o período de anestro em aproximadamente 40 dias, aumentando o percentual de prenhez ao final do acasalamento em $38 \%$.

Ao final do acasalamento, e também na desmama convencional, as vacas da CA280 apresentaram melhores escores de condição corporal $(\mathrm{P}<0,01)$ do que as vacas da CA360, tanto para vacas da desmama precoce quanto da desmama convencional (Tabela 1). O manejo de rebanhos de cria com carga animal acima da capacidade de suporte das pastagens tem sido a causa de baixa condição corporal e falhas reprodutivas (Osoro, 1989). Acredita-se que, agrupando as vacas, entre 90 e 100 dias antes do parto, por condição corporal, e conduzindo-as de modo que possam parir com condição corporal igual ou maior a 3, permitir-se-á obtenção de melhores índices reprodutivos.

Ao final do acasalamento, apenas 37 dias após a desmama precoce, as vacas deste tratamento já apresentavam uma diferença significativa em condição corporal, em ambas as cargas analisadas. Vacas mantidas em CA280 apresentaram escores de 3,46 e 4,09 , para as desmamas convencional e precoce, respectivamente, e vacas da CA360 apresentaram escores de 2,85 e 3,01, para as desmamas convencional e precoce, respectivamente $(\mathrm{P}<0,01)$. Essa variação foi devida, essencialmente, à grande diferença da oferta de forragem disponível aos animais nas cargas analisadas e à menor exigência em nutrientes de vacas desmamadas, permitindo, assim, maiores recuperações nas condições corporais. Santana \& Lobato (1983), em uma análise entre a desmama precoce e a desmama convencional, observaram, para vacas do primeiro grupo, condição corporal média de 3,54, contra 3,0, para as vacas do segundo grupo $(\mathrm{P}<0,05)$, na data da desmama convencional. Assim, além das cargas animais mais leves permitirem as vacas terem, na temporada de acasalamento, melhores condições corporais, a desmama precoce do bezerro é outro fator a permitir às vacas melhores recuperações de condições corporais no pós-parto, aumentando as chances de concepção durante o período reprodutivo.

Constam na Tabela 2 os percentuais de prenhez conforme as cargas animais, as composições raciais das vacas e idades à desmama. A composição racial das vacas não influenciou significativamente as taxas 
de prenhez observadas $(\mathrm{P}>0,05)$, embora as vacas $1 / 2$ Nelore $1 / 2$ Hereford tenderam a apresentar taxas numericamente mais altas $(47,06 \%$ vs. $41,86 \%)$. A CA280 permitiu índices de prenhez significativamente superiores $(\mathrm{P}<0,05)$ do que a CA360 $(67,56 \%$ vs. $22,56 \%)$. Segundo Scaglia (1997), a condição corporal das vacas, no acasalamento, afeta significativamente o desempenho reprodutivo, em termos do número de serviços necessários por concepção, intervalo de partos e percentual de vacas prenhes. Segundo o autor, condição corporal, ao início do acasalamento, menor ou igual a 2,5 (escala 1-5) resulta em taxas de prenhez extremamente baixas, sendo necessária melhor nutrição durante o acasalamento, para a obtenção de índices reprodutivos satisfatórios. Portanto, a carga animal mais baixa, como a estudada neste trabalho, pode permitir melhores recuperações de peso no pós-parto e condições corporais melhores, no período de acasalamento, possibilitando, com isso, melhores performances reprodutivas.

Simeone \& Lobato (1996) encontraram diferenças significativas na taxa de prenhez das vacas, conforme a carga animal em que as mesmas foram manejadas $(69,8$ e $51,7 \%$ para cargas animais de 240 e $340 \mathrm{~kg}$ PV/ha, respectivamente), tendo relacionado a melhor performance reprodutiva à menor carga animal e à melhor condição corporal das vacas, já ao início do acasalamento. Gottschall \& Lobato (1996), estudando os efeitos da carga animal sobre o comportamento reprodutivo de vacas primíparas Nelore x Devon, observaram índices de 8,5; 10,4 e $0,0 \%$, para cargas animais de 280,320 e $360 \mathrm{~kg} \mathrm{PV} / \mathrm{ha}$, respectivamente. Os autores concluíram serem estes baixos valores observados, resultado da baixa condição corporal e peso vivo ao parto, e dos ganhos de peso, ainda que bons no pós-parto, insuficientes para adequada recuperação de peso e condição corporal. Quadros \& Lobato (1996) obtiveram, em cargas animais de 240 e $320 \mathrm{~kg} \mathrm{PV} / \mathrm{ha}$, índices de prenhez de $96,8$ e $86,8 \%$ ( $P>0,05)$, respectivamente. A condição corporal, ao início do acasalamento, foi de 3,20 e 3,13 (escala 1-5) $(\mathrm{P}>0,05)$, para as cargas 240 e $320 \mathrm{~kg}$ $\mathrm{PV} /$ ha, respectivamente. Portanto, a condição corporal, ao início e durante o período de acasalamento, está relacionada estreitamente com a repetição de prenhez das vacas.

Na Tabela 2, são apresentados os percentuais de prenhez conforme a idade da desmama. Não houve diferença $(\mathrm{P}>0,05)$ na taxa de prenhez entre as vacas com bezerros submetidos a desmama precoce e desmama convencional e entre composições raciais das vacas.

Um dos fatores que podem ter contribuído para a inexistência de diferenças significativas na taxa de prenhez entre desmama convencional e desmama precoce pode ter sido o curto intervalo de tempo entre a data da desmama precoce e o final do acasalamento, pois da desmama precoce até a retirada definitiva dos touros decorreram apenas 37 dias. Lobato (1999) salienta ser o período de tempo entre a desmama precoce e o final do acasalamento uma importante fonte de variação no sucesso ou não da aplicação da desmama precoce. Também, o mesmo autor ressalta, em sua revisão, ser os resultados de prenhez com a desmama precoce variáveis, pois os mesmos são dependentes da condição corporal das vacas, quando da desmama.

Santana \& Lobato (1983) observaram, em rebanhos de três propriedades no Rio Grande do Sul, índices de prenhez de 90,$9 ; 86,4$ e $68,2 \%$, para vacas com bezerros desmamados aos 90 dias, enquanto naquelas com desmama aos 210 dias, os índices foram de 18,2; 54,5 e 4,5\%, respectivamente. Simeone \& Lobato (1996) relatam índices de prenhez de 94,1 e 40,3\% para vacas submetidas às desmamas à idade precoce e convencional de seus bezerros, respectivamente. Silva et al. (1995) reportam índices de prenhez de 54,0 e $27,0 \%$ para vacas primíparas submetidas aos tratamentos de desmama precoce (bezerros com

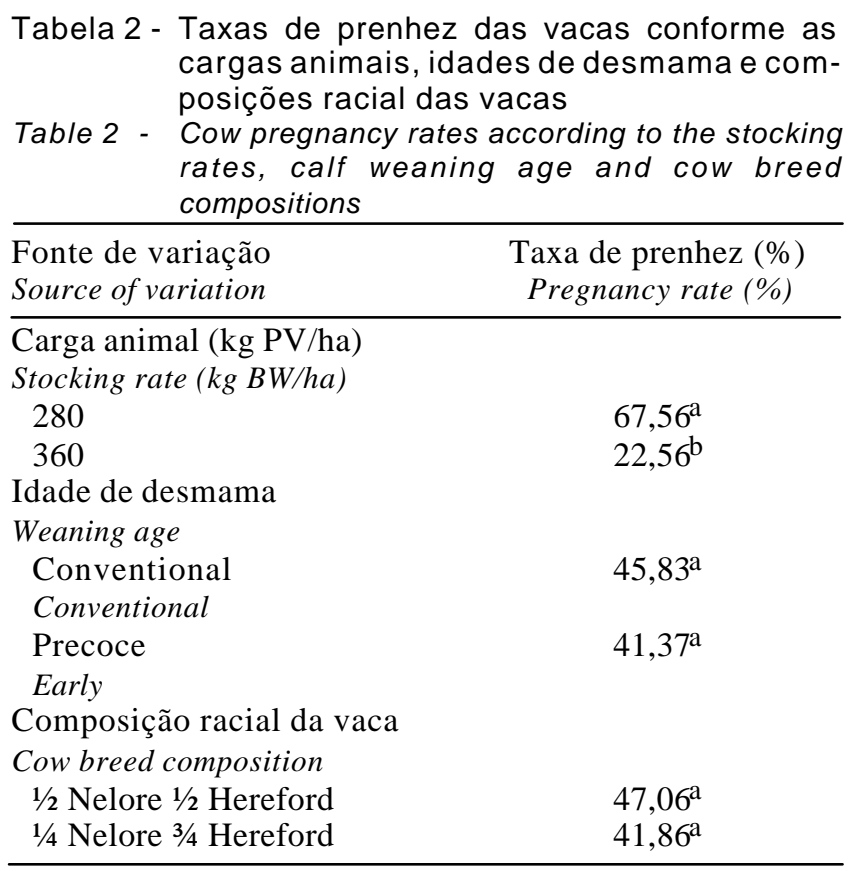


70 dias, em média) e desmama convencional (terneiros com 130 dias, em média), respectivamente, e para vacas adultas, índices de 75,0 e 53,0\% para desmama precoce e desmama convencional, respectivamente, sendo que, em ambos os casos, os índices diferiram entre si $(\mathrm{P}<0,05)$.

Como já mencionado, neste trabalho vacas $1 / 2$ Nelore $1 / 2$ Hereford tenderam numericamente a apresentar taxas de prenhez maiores que vacas $1 / 4$ Nelore $3 / 4$ Hereford (Tabela 2). Isto pode ser reflexo da melhor adaptação ao meio e rusticidade de fêmeas $1 / 2$ Nelore $1 / 2$ Hereford, em virtude de maior vigor híbrido desses animais (Teodoro \& Verneque, 1999).

Na Tabela 3, estão apresentados os resultados para IEP. A data juliana de parto (DJP) e a carga animal em que as vacas foram manejadas influenciaram o IEP $(\mathrm{P}<0,05)$. Para cada dia a mais na DJP, o IEP foi reduzido em 0,44 dias. Simeone \& Lobato (1996) observaram decréscimo de 0,99 dias no IEP, para cada dia de atraso do parto à estação de parição. Em condições distintas às do Rio Grande do Sul, Bourdon \& Brinks (1983) relataram redução de 0,86 dias no IEP, para cada dia de atraso do parto dentro da estação de parição.

Vacas da CA280 tiveram menor IEP que as vacas da CA360. Conforme discutido anteriormente, isto deve ser reflexo direto da maior oferta forrageira e, conseqüentemente, do melhor estado nutricional das vacas manejadas em carga animal de $280 \mathrm{~kg} \mathrm{PV} / \mathrm{ha}$. Esta maior disponibilidade forrageira permitiu às vacas retorno mais rápido à sua atividade reprodutiva. Magalhães \& Lobato (1991) também observaram menor IEP para vacas manejadas em 0,5 vaca com cria/ha do que com 0,7 vaca com cria/ha (384 vs. 427 dias, respectivamente). Simeone \& Lobato (1996) relatam, mesmo sem diferença significativa $(\mathrm{P}>0,05)$, IEP menor para vacas manejadas em carga animal de $240 \mathrm{~kg}$ PV/ha do que em $340 \mathrm{~kg}$ PV/ha (388 e 394 dias, respectivamente).

IEP próximos a 365 dias são desejáveis, a fim de que as vacas possam produzir um bezerro a cada ano. Segundo Orcasberro (1991), o IEP é um fator de grande importância dentro do manejo reprodutivo de rebanhos bovinos de corte, para que as vacas desmamem um bezerro a cada ano. Comparando o IEP observado neste trabalho com aquele do trabalho de Magalhães \& Lobato (1991) para vacas de parição à mesma idade e carga animal semelhante, observa-se menor IEP nesta pesquisa que o obtido por aqueles autores (395 vs. 427, respectivamente). IEP de 395
Tabela 3 - Intervalo de partos (IEP) das vacas, conforme as cargas animais, as idades de desmama dos bezerros e as composições raciais das vacas

Table 3 - Cow calving interval (Cl), according to the stocking rates, calf weaning ages and cow breed compositions

\begin{tabular}{|c|c|}
\hline $\begin{array}{l}\text { Fonte de variação } \\
\text { Source of variation }\end{array}$ & $\begin{array}{l}\text { IEP (dias) } \\
C I(\text { days })\end{array}$ \\
\hline \multicolumn{2}{|l|}{ Composição racial } \\
\hline \multicolumn{2}{|l|}{ Breed composition } \\
\hline $1 / 2$ Nelore $1 / 2$ Hereford & $402,2^{\mathrm{a}}$ \\
\hline $1 / 4$ Nelore $3 / 4$ Hereford & $403,2^{\mathrm{a}}$ \\
\hline \multicolumn{2}{|l|}{ Carga animal (kg PV/ha) } \\
\hline \multicolumn{2}{|l|}{ Stocking rates (Kg LW/ha) } \\
\hline 280 & $395,4^{\mathrm{a}}$ \\
\hline 360 & $409,9^{b}$ \\
\hline \multicolumn{2}{|l|}{ Idade de desmama } \\
\hline \multicolumn{2}{|l|}{ Weaning age } \\
\hline Convencional & $406,9^{\mathrm{a}}$ \\
\hline $\begin{array}{l}\text { Conventional } \\
\text { Precoce } \\
\text { Early }\end{array}$ & $398,4^{\mathrm{a}}$ \\
\hline $\begin{array}{l}\text { Data juliana de parto } \\
\text { Julian birth date }\end{array}$ & $0,44 *$ \\
\hline \multicolumn{2}{|c|}{$\begin{array}{l}\text { Médias seguidas de letras distintas, nas colunas, dentro de cada } \\
\text { fonte de variação, diferem significativamente }(P<0,05) \text { pelo F- } \\
\text { teste. } \\
\text { * Significante }(P<0,05) \text { pelo t-test. } \\
\text { Means followed by different letters in the column, within each source of } \\
\text { variation, are significantly different }(P<.05) \text { by F-test. } \\
\text { * Significant }(P<.05) \text { by t-test. }\end{array}$} \\
\hline
\end{tabular}

dias não é ideal, mas demonstra que o ajuste de carga animal para carga menor, com a conseqüente melhor of erta alimentar, permitiu melhores recuperação no pós-parto e desempenho reprodutivo das vacas.

A idade de desmama e a composição racial das vacas não apresentaram efeito significativo no IEP $(P>0,05)$. Lobato et al. (2000), no entanto, obtiveram IEP de 359 e 381 dias, para vacas primíparas, em pastagens melhoradas, no pré e pós-parto, submetidas à desmama precoce e convencional, respectivamente. Neste caso, a retirada antecipada dos bezerros, mesmo em pastagens melhoradas de ciclo hiberno/primaveril, permitiu às vacas antecipar, também, $o$ retorno à atividade reprodutiva.

\section{Conclusões}

A carga animal menor (280 kg PV/ha) permitiu maior desenvolvimento e acúmulo de forragem no campo nativo, disponibilizando, assim, maior quantidade de alimento às vacas.

A carga animal menor permitiu maiores recupe- 
rações de peso e condição corporal no pós-parto, possibilitando às vacas melhor condição corporal no início do acasalamento, melhores taxas de reconcepção e menores intervalos de partos.

A desmama definitiva dos bezerros em idade mais precoce (três/quatro meses) permitiu às vacas melhores recuperações de peso e condição corporal, porém não afetou significativamente a taxa de prenhez, o que enfatiza a necessidade de a desmama ser realizada em data que permita tempo suficiente para as vacas retornarem à atividade reprodutiva dentro da mesma estação.

\section{Literatura Citada}

BOURDON, R.M.; BRINKS, J.S. Calving date versus calving interval as a reproductive measure in beef cattle. Journal of Animal Science, v.57, n.6, p.1412-1417, 1983.

FREITAS, E.A.; LOPEZ, J.; PRATES, E.R. Produtividade de matéria seca, proteína digestível e nutrientes digestíveis totais em pastagem nativa do Rio Grande do Sul. Anuário Técnico IPZFO, v.3, p.454-515, 1976.

GOTTSCHALL, C.S.; LOBATO, J.F.P. Comportamento reprodutivo de vacas de corte primíparas submetidas a três lotações em campo nativo. Revista Brasileira de Zootecnia, v.25, n.1, p.46-57, 1996.

HANCOCK, K.L.; KROP, J.R.; LUSBY, K.S. et al. The influence of postpartum nutrition and weaning age of calves on cow body condition, estrus, conception rate and calf performance of fall-calving beef cows. Animal Breeding Abstract, v.53, n.1, p.932, 1985.

HARLAN, J.R. Generalized curves for gain per head and gain per acre in rates grazing studies. Range Management, v.11, n.1, p.140-147, 1958.

HART, R.H.; WAGGONER JR., J.W.; DUNN, T.G. et al. Optimal stocking rate for cow-calf enterprises on native range and complementary improved pasture.Range Management, v.41, n.5, p.435-441, 1988.

HAYDOCK, R.H.; SHAW, N.H. The comparative yield method for estimating dry matter yield of pasture. Australiam Journal of Experimental Animal Husbry, v.15, n.1, p.663, 1975.

HODGSON, J.; TAYLER, J.C.; LONSDALE, C.R. The relationship between intensity of grazing and herbage consumption and growth of calves. Journal of British Grassland Society, v.26, n.1, p.231-237, 1971.

LOBATO, J.F.P. Gado de cria tópicos. Porto Alegre: Adubos Trevo, 1985. 32p.

LOBATO, J.F.P. Considerações efetivas sobre seleção, produção e manejo para maior produtividade dos rebanhos de cria. In: LOBATO, J.F.P.; KESSLER, A.M.; BARCELLOS, J.O.J. (Eds.). Produção de bovinos de corte. 1.ed. Porto Alegre: PUCRS, 1999. p.286-302.

LOBATO, J.F.P.; BARCELLOS, J.O. Efeitos da utilização de pastagem melhorada no pós-parto e do desmame aos 100 ou 180 dias de idade no desempenho reprodutivo de vacas de corte. Revista Brasileira de Zootecnia, v.21, n.3, p.385-395, 1992.

LOBATO, J.F.P.; MÜLLER, A.; PEREIRA NETO, O.A. et al.
Efeitos da idade à desmama dos bezerros sobre o desempenho reprodutivo de vacas de corte primíparas. Revista Brasileira de Zootecnia, v.29, n.6, p.2014-2018, 2000.

LOWMAN, B.G.; SCOTT, N.; SOMERVILLE, S. Condition scoring beef cattle. Edingburgh: East of Scotland College of Agriculture (Bulletin 6), 1976. 8p.

MAGALHÃES, F.R.; LOBATO, J.F.P. Efeitos da utilização de pastagem e da idade ao primeiro parto nodesempenho reprodutivo de novilhas de corte. In: REUNIÃO ANUAL DA SOCIEDADE BRASILEIRA DE ZOOTECNIA, 28., 1991, João Pessoa.Anais... João Pessoa: Sociedade Brasileira de Zootecnia, 1991. p.424.

MONJE, A.; HOFER, C.; GALLI, I. Destet precoz. Efecto sobre los vientres, manejo menejo de los terneros e impacto de la tecnica sobre los sistemas de produccion. In: JORNADA DE DIFUSIÓN TECNICA, 1., 1993. Destet precoz en cria vacuna. Concepción del Uruguai: INTA. Centro Regional Entre Rios, 1993. 59p.

MOTT, G.O. Grazing pressure and the measure of pasture production. In: INTERNATIONAL GRASSLAND CONGRESS, 8., 1980, Pensylvania. Proceedings... Pensylvania: State College Press, 1980. p.606-611.

NICOL, A.M.; NICOLL, G. B. Pastures for beef cattle. In: NICOL, A.M. (Ed.). Feeding livestock on pasture.Lincoln: New Zeland Society of Animal Production, 1987. p.110-131, (Occasional publication, 10).

ORCASBERRO, R. Estado corporal, control de amamentamiento y performance reproductiva de los rodeos de cria. In: PASTURAS Y PRODUCCIÓN ANIMAL EN ÁREAS DE GANADERIA EXTENSIVA. Resumen... Montevideo: INIA, 1991. p.12-16. (Série técnica, 13)

OSORO, K.O. Efecto de las principales variables de manejo sobre los parametros reproductivos en las vacas de cria. Producción y Sanidadd Animal, v.1, n.1-2, separata n. 7, p.87-111, 1986.

OSORO, K.O. Manejo de las reservas corporales y utilización del pasto en los sistemas de producción de carne com vacas madres estabelecidos en zonas húmedas. Producción y Sanidad Animal, v.4, n.3, p.6-88, 1989.

QUADROS, S.A.F.; LOBATO, J.F.P. Efeitos da lotação no comportamento reprodutivo de vacas de corte primíparas. Revista Brasileira de Zootecnia, v.25, n.1, p.23-35, 1996.

RICHARDS, M.W.; WETTEMANN, R.P.; SCHOENEMANN, H.M. Onset of anestrus in nutritionally restricted Hereford cows. Journal of Animal Science, v.63, n.2, p.62-70, 1989.

SANTANA, G.A.O.; LOBATO, J.F.P. Efeitos de diferentes pesos e idades na desmama no desenvolvimento de terneiros e comportamento reprodutivo de vacas de corte. Eficiência reprodutiva. In: REUNIÃO ANUAL DA SOCIEDADE BRASILEIRA DE ZOOTECNIA, 20., 1983, Pelotas. Anais... Viçosa, MG: Sociedade Brasileira de Zootecnia, 1983. p.227.

STATISTICAL ANALYSIS SYSTEM -SAS. SAS/STAT: User's Guide. version 6. 4.ed. Cary: 1996.

SCAGLIA, G. Nutricion y reproduccion de la vaca de criaUso de la condición corporal. Paysandú: INIA, 1997. v.91, 16p. (Série tecnica)

SILVA, M.D.; BECKER, C.; LOBATO, J.F.P. Efeito do desmame aos 60 dias na taxa de prenhez de vacas de corte primíparas e de parto tardio. In: SALÃO DE INICIAÇÃO CIENTÍFICA, 7., 1995, Porto Alegre. Resumos... Porto Alegre: Universidade Federal do Rio Grande do Sul, 1995. p.97. 
SIMEONE, A.; LOBATO, J.F.P. Efeitos da lotação animal em campo nativo e do controle da amamentação no comportamento reprodutivo de vacas de corte primíparas. Revista Brasileira de Zootecnia, v.25, n.6, p.1216-1227, 1996.

STEEL, R.G.D.; TORRIE, J.H. Bioestadística: principios y procedimientos. Mexico, McGraw Hill, 1989. 622p.

TEODORO, R.L.; VERNEQUE, R.S. Sistema de cuzamento como alternativa para o melhoramento de bovinos. In: REUNIÃO ANUAL DA SOCIEDADE BRASILEIRA DE ZOOTECNIA, 36., 1999, Porto Alegre. Anais... Porto Alegre: Sociedade Brasileira de Zootecnia, 1999. p.241-249.

Van SOEST, P.J. Nutritional ecology of the ruminant. Corvalis: O \& P Books, 1982. 374p.
WRIGHT, I.A.; RHIND, S.M.; RUSSEL, A.J.F. et al. Effects of body condition, food intake and temporary calf separation on duration of the post-partum anoestrus period and associated LH, FSH and prolactin concentrations in beef cows.Animal Production, v.45, n.2, p.1049-1055, 1987.
Recebido em: 09/07/02

Aceito em: 19/03/03 